\title{
Wireless Gesture Controlled Semi-Humanoid Robot
}

\author{
Toshika Fegade, Yogesh Kurle, Sagar Nikale, Praful Kalpund
}

Mumbai University, India

\begin{tabular}{|c|c|}
\hline Article Info & ABSTRACT \\
\hline Article history: & \multirow{10}{*}{$\begin{array}{l}\text { A Robotics is a field concerned with the "intelligent connection of perception } \\
\text { of action". The most common manufacturing robot is the robotic arm with } \\
\text { different degree of freedoms. Today, these humanoids perform many } \\
\text { functions to assist humans in different undertakings such as space missions, } \\
\text { driving and monitoring high speed vehicles. They are called Humanoids } \\
\text { because they resemble humans. The idea of this paper is to change perception } \\
\text { of controlling robotic arm. This paper provides a way to get rid of old } \\
\text { fashioned remote controls and gives an intuitive technique for } \\
\text { implementation of Semi-Humanoid Gesture controlled robot. The motion } \\
\text { sensor used are -flex and accelerometer (used in mobile phones for tilting } \\
\text { motion). It includes two robotic arms which are exactly similar to human } \\
\text { arms (with } 5 \text { fingers), increasing sensitivity of the system, a moving platform } \\
\text { and a camera for capturing real time video. The prime aim of the system is } \\
\text { "to design a proposed system in such a way that user can operate the robot } \\
\text { with the ease through gestures". This involves movement of both robotic } \\
\text { arms \& platform in synchronous with user's hand \& leg gestures } \\
\text { respectively. }\end{array}$} \\
\hline Received Sep 19, 2016 & \\
\hline Revised Oct 28, 2016 & \\
\hline Accepted Nov 10, 2016 & \\
\hline & \\
\hline Keyword: & \\
\hline Index Terms - flex sensor & \\
\hline Accelerometer & \\
\hline Degree of freedom (DOF) & \\
\hline Humanoid & \\
\hline
\end{tabular}

Copyright $(0) 2016$ Institute of Advanced Engineering and Science. All rights reserved.

\section{Corresponding Author:}

Toshika Fegade, Mumbai University, India.

Email: toshikafegade@gmail.com

\section{INTRODUCTION}

A robotic arm is a mechanical arm made of aluminium material which can be controlled with the use of electronic motors. Considering the use of remotes, it is very difficult to control multiple keys at the same time and it also requires to analyse 3D projected motion. It requires a fine judgement or a lot of practice. So to avoid such inconvenience is the star of the article to make the existing system more intuitive and user friendly.

Gesture recognition technologies are much younger in the world of today. At this time there is much active research in the field and little in the way of publicly available implementations. Glove based technique is a well-known means of recognizing hand gestures. It utilizes a sensor attached to a glove that directly measures hand movements. The user just needs to wear a small transmitting device on hand which includes a sensor which is an accelerometer and flex sensor in this case. An Arduino is used in this project as control unit.

The system is equipped with 2 cameras up front in place of eyes which can stream real time video wirelessly to any Laptop. The Robot is mounted over a movable platform which is also controlled wirelessly.

\section{RELATED WORK}

Today, there are a number of robotic arms used in robotics research and industrial applications. This robotic arms can be defined or trained for certain applications and designed that way particularly. With the use of gesture control, the controlling part becomes simple and effective. In a generalized manner, most of these can be categorized into either of the following two models: 


\subsection{Model 1}

The arm can move up, down, rotate towards right or left. But the main limitation of this is, it cannot work as a human hand. It can only place and pick up the objects with lesser efficiency.

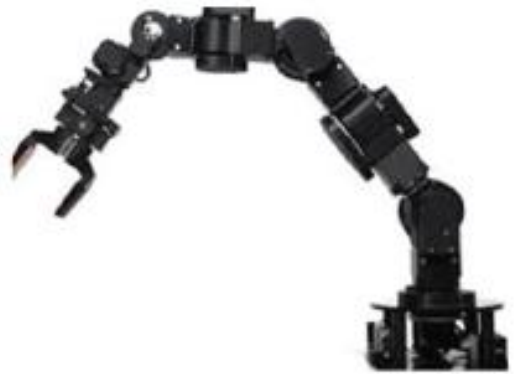

Figure 1. Model 1-Pick and place [8]

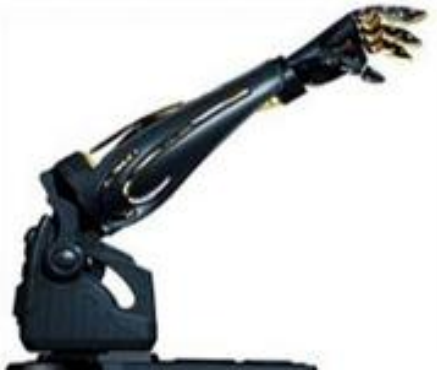

Figure 2. Model 2-Stationary [9]

\subsection{Model 2} stationary.

It will be more efficient as it can acts perfectly like a human hand. But its main limitation is, it is

According to the existing literature the models of the robotic arm have been observed to be stationary and the others were consisting of grippers which were not so efficient when it came to picking up objects. Basically the idea is to negotiate the limitations from the existing systems and to design and implement the model while considering the following objectives:

- Robotic arms having functionalities of human arms including human like fingers.

- Mounted on a platform having 4 wheels for movements.

- A video camera which enables the user to see what the robot is seeing.

- 19 DOF per hand.

First Degree: Up and down movement of shoulder which is known as pitch.

Second Degree: The movement of elbow in up and down position which is known as elbow pitch.

Third Degree: side to side movement of the elbow

Fourth Degree: The movement of wrist in up and down position known as wrist pitch

Fifth Degree: The movement of wrist in a rotating position known to be wrist roll.

Fingers: 3 DOF (per) finger and 2 DOF for thumb

\section{MOTIVATION}

Bomb squad officials, soldiers risk their life while working for the nation. Many complex and contagious medical surgeries can be performed with help of humanoid robotic arm. Also, paralyzed patients can be made independent if they are taught to use this system effectively. Scanning and verification of official documents is another added feature with01 image processing.

For several decades already, industrial robots have proved their value by obtaining high performance in terms of speed, accuracy and reliability. Robots are built that even have nanometre precision. The constructions are very stiff, the behavior is almost perfectly linear and every degree of freedom can independently be controlled. Moreover, the operating environment of the robots is known a-priori and the number of objects is limited. This way, relatively simple control techniques can be used to make exact positioning possible. The main inspiration for this system is 'Robo Sally[10][11][12], a bomb disposal robot developed and used by U.S. Defense. With the use of upcoming technology and by adding multiple features, a user-friendly system is developed.

\section{PROBLEM DEFINITION}

Life of a human being is the most precious thing. Still bomb squad officials lose their life defusing bombs, manning checkpoints, soldiers lose their life saving the other injured soldier, doctors \& nurses risk their life examining contagious patients, and scientist risk their life working with highly radioactive elements. Several precautions are taken, still many people lose their life. However all these jobs can be efficiently done by a Robot if controlled by a professional. Gesture Controlled Semi-Humanoid Robot will help to get over these problems without risking the precious life of people and making them independent. 


\section{PROBLEM STATEMENT}

In the industry the robots are used as a tool to facilitate the work so it is necessary for them to satisfy some criteria. There are main problem that arise in the existing robot arm:

Difficult to control and time consuming to control.

The project proposed in this paper is having upper body similar to human body with 2 arms and lower body is replaced by a platform, hence called semi-humanoid. It can be controlled by corresponding gestures of operator's hand and leg, exactly working as a human robot, making it easier to control and fast in operation.

\section{TECHNICAL REQUIREMENTS}

\subsection{Microcontroller}

The controlling units used here are basically Arduino mega [4] \& Arduino Uno. The robotic arms use Arduino Mega while the platform assembly uses Arduino Uno. The microcontroller used in the Arduino Mega is ATmega2560 \& that of Arduino Uno is ATmega 328P. For each robotic arms two Arduino Megas are required .One at transmitting end \& other at receiving end. Similarly platform assembly requires two Arduino Unos. In total we require 6 Arduinos boards.

\subsection{Flex sensor ${ }^{[5]}$}

They are passive resistive devices which gives variable resistance depending on the bending radius. They are available in various format depending on the type of the material used, the size of the sensor $\&$ the application. In our project, we used Conductive Ink-based, 2.2 inch, unidirectional flex sensor. We use 1 flex sensor for each finger at the transmitting end.

\subsection{Accelerometer Sensor ${ }^{[6]}$}

The ADXL335 ${ }^{[6]}$ is a small, thin, low power, complete 3- axis accelerometer with signal conditioned voltage outputs. Total 7 accelerometers used in this project are ADXL335. For each hand, we require one accelerometer for shoulder movement, one for elbow movement, one for wrist movement and the remaining one is used for platform movement.

\subsection{Rf module ${ }^{[7]}$}

RF stands for Radio Frequency. This module consists of further two parts: Transmitter (Tx) and Receiver $(\mathrm{Rx})$. It is available in different operating frequencies with different operating range. An RF (radio frequency) module operating at $433 \mathrm{MHz}$ frequency is used to transmit and receive radio signals between user (transmitter) and the robot (receiver). One RF module is used per each hand \& one is used for platform assembly. So total 3 RF module pairs are used.

\section{PROPOSED SYSTEM}

In this paper, a robotic arm with 19 degrees of freedom (per arm) is designed. The system is categorized into the following parts:

- Robotic arms

- Platform assembly

- RF communication

- Wireless video transmission

\subsection{Robotic Arm}

The heart of this project is the two robotic arms. The structure of this arm is similar to human arm. It is developed in such a way that the complex movements such as shoulder up down, elbow up-down \& rightleft, wrist up-down \& rotation. Fingers movement is the additional feature of this system which is rarely observed in the other robotic arms.

The arm is equipped with gesture synchronized servo motors. The flex sensors (mounted on fingers) and accelerometer (mounted on wrist, elbow and shoulder) captures the hand gestures. At the transmitting end RF transmitter module is interfaced to the control unit (Arduino) which senses the motion of human gestures and transmit it to the robot. At the receiving end RF Receiver module will receive the data and this data is then processed by a control unit and passed on to rotate the motors in a special configuration to make the robot move in the same direction as that of the human gestures. 


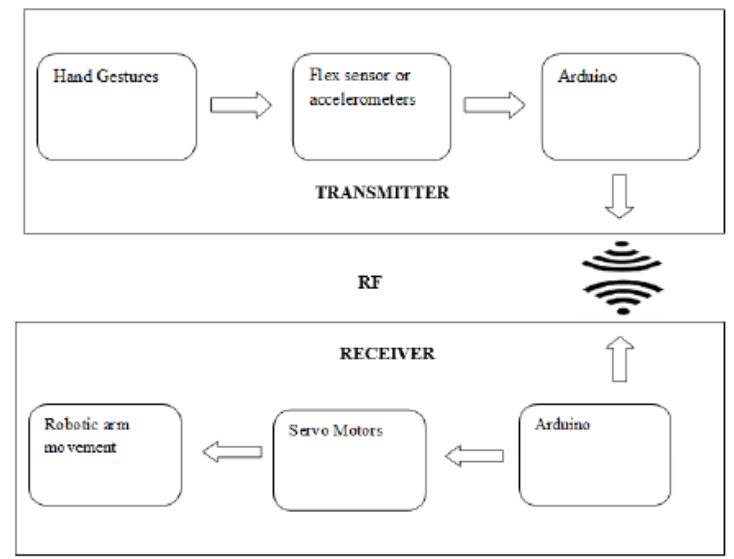

Figure 3. Hand Movement Flowchart

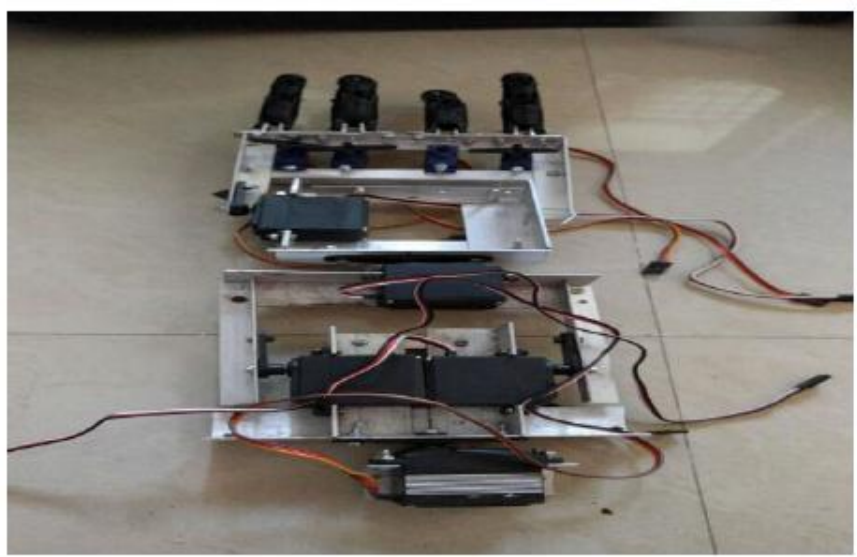

Figure 4. Hand assembly

\subsection{Platform Assembly}

The robotic arms are mounted on the platform assembly. This assembly has a wooden platform with 4 DC motors which are capable of handling the load of whole robot. An accelerometer is placed on one of the user's leg. Depending on the leg gestures a value is sent to the controlling unit (Arduino Uno) which is situated on the platform assembly enabling the corresponding platform motion. Gestures are- Forward, Backward, Right, Left. Block Diagram:

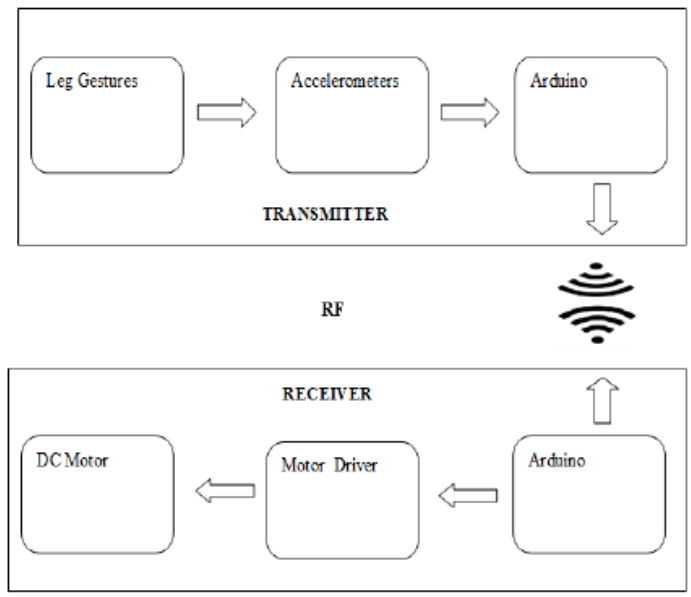

Figure 5. Platform Movement 


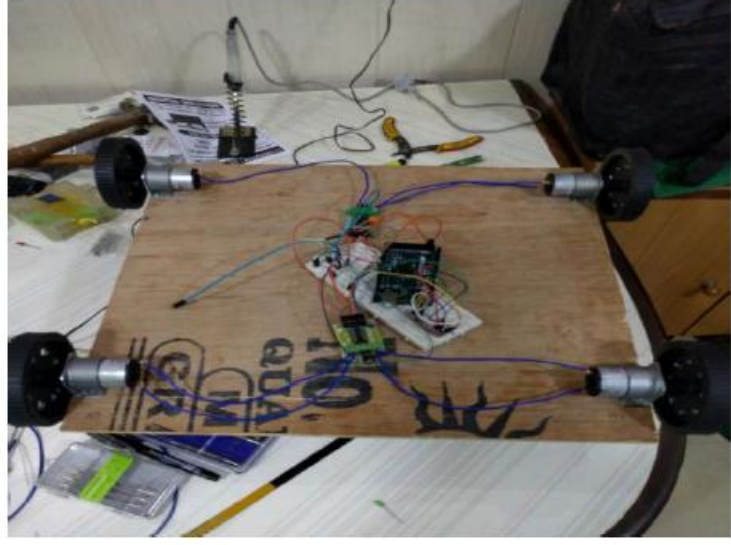

Figure 6. Platform

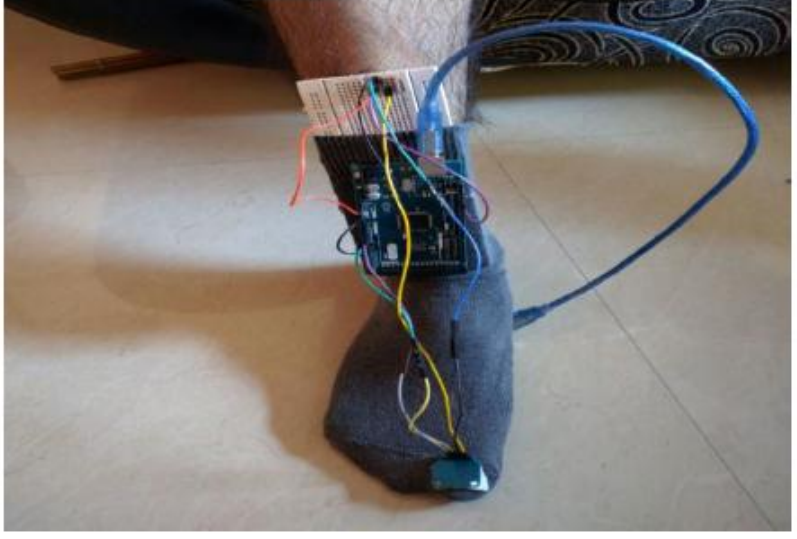

Figure 7. Gesture control for Platform

\subsection{RF Communication}

An RF module is used as a medium for communication between user and robot.

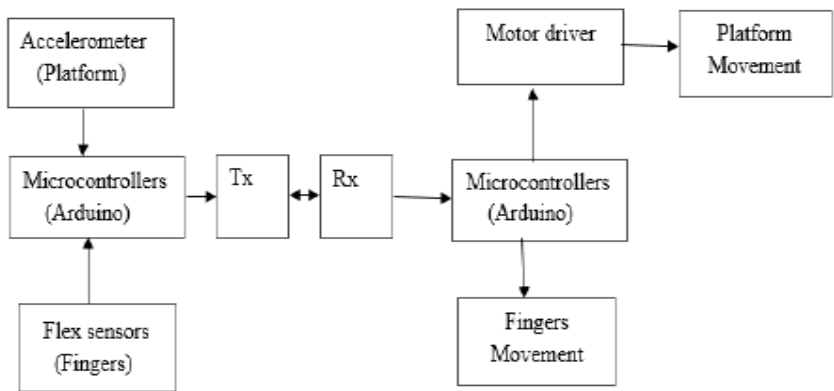

Figure 8. Block diagram for communication system

\subsection{Wireless Video Transmission}

A camera module is used as a robot eye enabling the user to see what the robot is actually seeing. The information captured by the camera is sent wirelessly to the user's mobile or laptop.

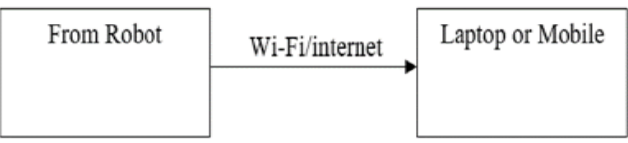

Figure 9. Video assembly

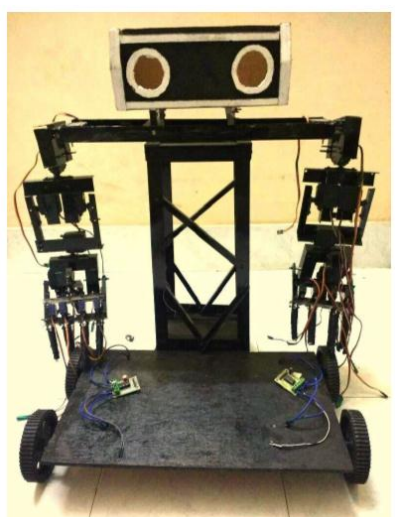

Figure 10. Robot structure 


\section{RESULTS}

\subsection{Platform}

\begin{tabular}{|c|c|c|}
\hline Gesture & \multicolumn{2}{|c|}{ Accelerometer Readings } \\
\hline Forward & $>315$ & \multirow{2}{*}{ Z value } \\
\hline Backward & $<280$ & \\
\hline Right & $<310$ & \multirow{2}{*}{ X Value } \\
\hline Left & $>360$ & \\
\hline
\end{tabular}

Explanation:

The range for accelerometer values are tabulated for particular observation. The platform moves forward, backward, right and left with respect to the readings of accelerometer.

\subsection{Right Hand}

\begin{tabular}{|c|c|c|c|}
\hline $\begin{array}{l}\text { RIGHT } \\
\text { HAND }\end{array}$ & MOV1 & MOV2 & MOV3 \\
\hline Angle $x$ & $65-70$ & $45-55$ & $25-30$ \\
\hline Angle $z$ & $60-65$ & $52-60$ & $22-28$ \\
\hline Angle $x 1$ & $12-20$ & $32-42$ & $58-63$ \\
\hline Angle $z 1$ & $20-28$ & $17-26$ & $66-73$ \\
\hline
\end{tabular}

Explanation:

The accelerometer values are converted into angle values (spherical coordinates) for the ease of operation. Angle X,Y,Z stands for shoulder accelerometer and angle X1,Y1,Z1 for wrist accelerometer. The respective values for moves are tabulated that gives range of operation. Move 1 is 0 o in vertical plane. Move 2 is 90 o in vertical plane and move 3 is 180 o in vertical plane.

\subsection{Left Hand}

\begin{tabular}{|l|l|l|l|}
\hline LEFT HAND & MOV1 & MOV2 & MOV3 \\
\hline Angle x & $59-65$ & $42-48$ & $21-30$ \\
\hline Angle y & $43-49$ & $46-52$ & - \\
\hline Angle z & $64-70$ & - & - \\
\hline Angle x1 & - & $46-52$ & - \\
\hline Angle y1 & - & $47-52$ & - \\
\hline Angle z1 & $20-26$ & $56-62$ & - \\
\hline
\end{tabular}

Explanation:

The accelerometer values are converted into angle values(spherical coordinates) for the ease of operation. Angle X,Y,Z stands for shoulder accelerometer and angle X1,Y1,Z1 for wrist accelerometer. The respective values for moves are tabulated that gives range of operation. Move 1 is 0 o in vertical plane. Move 2 is 90 o in vertical plane and move 3 is 180 o in vertical plane.

\section{CONCLUSION}

Gesture control is the most simple and easy to use method. Multiple applications with limited number of gestures with high accuracy and sensitivity is verified. Simple hardware with microcontroller and sensors are used to develop the technology. In this, paper has reviewed the characteristics of the main mechanical structure and construction of a semi-humanoid robot. The final step of this robot is auto learner, in this stage this robot can learn automatically. The assistive robotic arm will must be able to contribute most of the challenges in our daily life. 


\section{FUTURE SCOPE}

The proposed design can be developed by making it path finder using neural network technology, image processing functions, face recognition, scanning, learning it for a particular application using neural network and artificial intelligence, for security. GPS system can be added to enable system to find location or to make a conference call. System can work at the places where human cannot step in.

\section{REFERENCES}

[1] Wan Faizura Binti Wan Tarmizi, Irraivan Elamvazuthi and Mumtaj Begam, " Kinematic and Dynamic Modeling of a MultiFingered robot Hand", vol 9, 2009.

[2] Md. Anisur Rahman , Alimul Haque Khan , Dr. Tofayel Ahmed , Md. Mohsin Sajjad, ”Design, Analysis and Implementation of a Robotic Arm- The Animator", vol 2, 2013

[3] Aakash k. Sancheti, "Gesture Actuated Robotic Arm", vol 2, 2012

[4] https://www.arduino.cc/en/Main/arduinoBoardMega

[5] http://www.engineersgarage.com/contribution/interfacing-flex-sensor-arduino

[6] http://playground.arduino.cc/Main/MPU-6050

[7] https://www.sparkfun.com/datasheets/Components/SMD/adxl335.pdf/

[8] http://www.energid.com/experience/low-cost-dexterous-robots/

[9] http://iq.intel.com/how-brain-controlled-robotic-arms-can-change-healthcare/

[10] http://www.engineering.com/DesignerEdge/DesignerEdgeArticles/Articl Johns-Hopkins-Researchers.aspx

[11] https://www.xsens.com/customer-cases/robo-sally-bomb-disposal-robot/

[12] http://www.jhuapl.edu/newscenter/stories/st080703.asp 